\title{
COGNITIVE FACTORS AND GENDER RELATED TO SELF-REPORTED DIFFICULTIES IN OLDER DRIVERS
}

\author{
Inês S. Ferreira, ${ }^{1}$ José F. Marmeleira, ${ }^{2}$ Mário B. Godinho, ${ }^{3}$ Mário R. Simões ${ }^{1}$ \\ ${ }^{1}$ Faculdade de Psicologia e de Ciências da Educação \\ University of Coimbra, Portugal \\ E-mail: ines.rsferreira@gmail.com \\ ${ }^{2}$ Departamento de Desporto e Saúde \\ University of Évora, Portugal \\ ${ }^{3}$ Faculdade de Motricidade Humana \\ Technical University of Lisbon, Portugal
}

\begin{abstract}
Summary: The purpose of this study is to determine the association between selfreported driving difficulties among older drivers and scores on specific cognitive tests. A cross-sectional study of 38 car drivers (22 men and 16 women), aged 6181 was carried out. The participants were volunteers recruited from two senior universities and a university health program for older drivers, in the area of Lisbon, Portugal. A battery of visual, psychomotor and cognitive measures was undertaken with a questionnaire about driving difficulties in a sample of healthy older adults. Difficulties in driving were the dependent variable in this study, defined as any reported difficulty in $\geq 4$ driving situations. After a multivariate analysis, the independent factors significantly associated with driving difficulties were divided attention subtest of $\operatorname{UFOV}^{\circledR}(p=0.013)$, number of trials with success in the Tower of London $(p=0.015)$ and gender $(p=0.021)$. The drivers that reported difficulties in $\geq 4$ driving situations made significantly less trials with success in the Tower of London test, had lower performances in divided attention between central and peripheral targets, and were more frequently females. Results suggest that planning ability, divided visual attention and gender are three important factors to be considered in the analysis of driving difficulties. However, further investigation with additional cognitive tests and driving measures (staterecorded crashes, driving simulator and on-road driving performance) could be developed in order to understand the factors related to driving difficulties in healthy older drivers.
\end{abstract}

\section{INTRODUCTION}

The proportion of elderly people in the population is increasing in the whole industrialized world, as well as the number of older drivers on the road. The development of methods to identify older driver problems has thus become an important research issue. One of the major challenges has been to understand the cognitive changes in the elderly that lead to impaired driving performance and high crash risk. Some progress has been made in identifying cognitive tests with predictive value in relation to driving performance or crash involvement in older drivers. These include: tests of visual attention including Useful Field of View (UFOV $\left.{ }^{\circledR}\right)(B a l l$ et al., 2006; Owsley et al., 1998; Whelihana et al., 2005) and Trail Making Test, part A (Kantor et al., 2004; Lundberg et al., 1998; 2003); tests of visual-constructional abilities including Block Design subtest from the WAIS (Lundberg et al., 1998; 2003) and Motor-free Visual Perception 
Test (Ball et al., 2006; Lundberg et al., 2003); tests of visual and verbal memory including ReyOsterrieth Complex Figure Test and Auditory Learning Verbal Test (Lundberg et al., 1998; 2003), respectively; tests of executive functioning including Tower of London (Daigneault et al., 2002) and Trail Making Test, part B (Ball et al., 2006; Kantor et al., 2004; Lundberg et al., 2003; Richardson \& Marottoli, 2003; Whelihana et al., 2005), among others.

Another major subject in current research is the driving difficulties of older drivers, mostly analysed by two questions: (1) which driving circumstances do they commonly avoid? (2) in which crash situations are they overrepresented? The first question is usually studied using questionnaires, relying on subjects' self-report; the second one looks to crash-recorded data. Research has shown that older drivers with poorer cognitive and/or visual function tend to limit their exposure to driving situations that are generally believed to be more difficult (e.g., rain, night, heavy traffic, rush hour) (Ball et al., 1998; Owsley et al., 1999). On the other hand, Maycock (1997) reviewed the available research on older drivers and the circumstances and features of accident involvement, concluding that older drivers find the following situations difficult: those that take place at junctions and intersecting traffic streams, those that involve right of way decisions, and those that require response to signs and signals.

Even in normal aging there is a decline in many cognitive abilities that can bring some driving difficulties. Usually, a decline in information processing speed, loss of efficiency in acquiring new information, cognitive inflexibility, and reduction in working memory function is indicated (Ball et al., 2004). However, the literature doesn't seem to clearly show whether cognitive factors are associated with the report of driving difficulties in older adults. The purpose of this study was to determine the association between self-reported driving difficulties rates in older people and scores on specific cognitive tests. In an attempt to achieve this goal, a battery of functional measures was undertaken using a questionnaire about driving difficulties in a sample of healthy older adults. This sample was chosen because poor mental status has been associated with lack of insight and, consequently, under-reporting of driving difficulties (Wild \& Cottrell, 2003). It was hypothesized that older drivers reporting more driving difficulties would be more likely to have lower scores on cognitive tests.

\section{METHOD}

\section{Participants}

A cross-sectional study of 38 drivers (22 men and 16 women) from 61 to 81 years old $(M=70.2$, $S D=5.0)$ was carried out. The participants were volunteers recruited from two senior universities and a university health program for older drivers, in that area of Lisbon, Portugal. All participants were still living independently in the community and did not constitute a specific clinical group. The exclusion criteria were: aged 60 years or younger; crash involvement in the last 2 years; cognitive impairment in Mini-Mental State Examination (MMSE) (Folstein et al., 1975) score; severe chronic illness; alcohol and drug dependence; or sensorial, motor or language deficit that could interfere on cognitive test results. All participants were currently driving ( $\geq 2$ days a week) and had had a driver's license for over 10 years. As to educational level, 26\% had completed elementary school, 32\% middle and high school and $42 \%$ were university graduates. 


\section{Procedure and Measures}

This study was conducted simultaneously with another investigation concerning time-to-arrival and useful field of view in older drivers. The evaluations were performed individually in the universities where the participants were recruited. All testing took place in a single session lasting 2 hours. Prior to participation, the study was explained and written informed consent was obtained from each participant. Participants were assured that the study results were confidential and had no bearing on their driving licence. To check inclusion criteria, a questionnaire was designed to gather information on demographic variables (age, gender, place of residence, education level, etc.), functional impairments and medical conditions (e.g., chronic conditions, alcohol and drug dependence, medications). Cognitive status was evaluated using the MMSE, a screening measure of global cognitive impairment. The study protocol included a battery of visual, physical and cognitive tests, and a survey on driving habits and difficulties. This questionnaire was adapted from previous research on older drivers (Owsley et al., 1999) and translated into Portuguese (translate - translate back method), adding 22 items concerning difficulties in driving situations in the last year. To explore the link between battery factors and reporting of driving difficulties, difficulty with driving was the dependent variable in this study, defined as any reported difficulty in $\geq 4$ driving situations. For data analysis, a bivariate analysis was made; subsequently, the variables associated with the difficulties in driving were submitted to multivariate analysis through a logistic regression, controlling the effects of age, education level and visual acuity. For all statistical analysis significance level was set at $p<0.05$.

\section{Protocol}

\section{Visual acuity}

Snellen Chart. With respect to visual function, distant visual acuity was measured monocular and binocularly with habitual correction at 6 meters.

\section{Physical Measure}

Foot Tap. While remaining seated, the participant was required to touch the floor on alternating sides of a 2-inch tall barrier 5 times with their right foot. Time (seconds) to complete is measured. This task assesses lower limb mobility, which was associated with the occurrence of adverse driving events (Marottoli et al., 1994).

\section{Cognitive Measures}

A cognitive assessment procedure was carried out, including UFOV ${ }^{\circledR}$ (Ball \& Owsley, 1993) and a battery of neuropsychological tests that cover several cognitive domains (Lezak et al., 2004; Strauss et al., 2006), such as: Trail Making Test (TMT-A \& TMT-B; Reitan, 1958); Tower of London (TOL; Shallice, 1982); Rey-Osterrieth Complex Figure Test (CFT-copy \& CFT-recall; Rey, 1941); Block Design subtest from the WAIS-III (BD; Wechsler, 1997). All tests were selected from previous research showing a relationship with driving measures (viz. crash involvement or on-road driving performance). 
1. UFOV ${ }^{\circledR}$ Test. This computer-administered and -scored test measures the visual field area over which one can use rapidly presented information, and is composed of three subtests: speed (in $\mathrm{ms}$ ) of visual processing, divided attention and selective attention. Several studies on older drivers support that impairment in the useful field of view is associated with crash involvement (e.g., Ball et al., 2006; Owsley et al., 1998) or on-road driving performance (e.g., Whelihana et al., 2005).

2. Trail Making Test. The participant uses a pencil to sequentially connect integers in ascending order (part A), or a mix of integers and letters in alternating and ascending order (part B). They are instructed to do the task as quickly as possible and without lifting the pencil from the paper. The time in seconds to finish each part is recorded. This test is used to assess speed of visual search, divided attention, sequencing with a motor component, and mental flexibility (Strauss et al., 2006). In particular, TMT-B is more sensible to executive functions (Lezak et al., 2004). Several studies on older drivers have demonstrated a correlation between TMT and crash involvement (e.g., Lundberg et al., 2003) or on-road driving performance (e.g., Kantor et al., 2004).

3. Tower of London. Participants are asked to preplan mentally a sequence of moves to match a start set of 3 coloured balls to a goal, and then to execute the moves one by one. The principal measures employed were: the preplanning time (time between the beginning of the task and the first movement), the execution time (total time minus planning time), the number of trials solved in the minimum movements possible and the number of instruction failures (the number of times the subject does not respect test instructions). This test is used to assess executive functions, in particular, planning ability (Lezak et al., 2004). In Daigneault et al. (2002) study, Tower of London test performance was associated with elderly drivers having a history of accidents.

4. Rey-Osterrieth Complex Figure Test. Participants are required to copy a complex geometric figure (copy task) before recalling it from memory some 3 minutes later (recall task). The quantitative correction is based on time (seconds), number and localization of the elements. Copy task provides a reliable index of visuoconstructional ability, while recall task measures visual memory (Lezak et al., 2004). In older drivers, both tasks were associated with crash involvement (Lundberg et al., 2003).

5. Block Design. The participant is asked to replicate a maximum set of 14 printed twodimensional geometric patterns using two-colour cubes. Four designs must be completed within 30 seconds, five within 60 seconds, and five within 120 seconds. A design can be failed because of faulty construction or exceeding the time limit. This task is a measure of visuoconstructional ability that correlates with performance IQ (Lezak et al., 2004) and was associated with crash involvement in older drivers (Lundberg et al., 1998, 2003).

\section{Self-reported Driving Habits and Difficulties}

Driving Habits Questionnaire (DHQ). This questionnaire was interviewer-administered to obtain driving information for the past year (for details see Owsley et al., 1999). The DHQ addresses six domains; the domain of interest in this study is driving difficultly. Additionally, 22 items were included about difficulties in driving situations (e.g., busy intersections, seeing signs in 
time to respond, passing other cars, judging speed and distance of oncoming cars, perceiving that front car has slowed down or stopped), in which participants had to respond "yes" or "no" if the statement applied to them. Information was also collected on driving exposure (days/week; $\mathrm{km} /$ week).

\section{RESULTS}

Several variables were associated with driving difficulties in the bivariate analysis. After the multivariate analysis, controlling the effects of age, education level and visual acuity, the independent factors significantly associated with driving difficulties were divided attention subtest of $\mathrm{UFOV}^{\circledR}(p=0.013)$, number of trials with success in the Tower of London $(p=0.015)$ and gender $(p=0.021)$ (Table 1). The drivers that reported difficulties in $\geq 4$ driving situations made significantly less trials with success in the Tower of London test, had lower performances in divided attention between central and peripheral targets, and were more frequently females.

Table 1. Descriptive statistics for the variables associated with reported difficulties in driving; $p$-values for those variables in the multivariate model (logistic regression)

\begin{tabular}{lrrrr}
\hline & \multicolumn{3}{c}{ Descriptive Statistics } & \\
\cline { 2 - 4 } & $\%$ & Mean & SD & $p^{{ }^{a}}$ \\
\hline Gender & & & & $0.021^{*}$ \\
Male & 57.9 & & & \\
Female & 42.1 & & & \\
Age & $70.18 \pm 4.96$ & 0.965 \\
FootTap (sec) & $5.55 \pm 1.20$ & 0.962 \\
Subtest 2 of UFOV ${ }^{\circledR}$ (msec) & $171.0 \pm 134.1$ & $0.013^{*}$ \\
TMT-A (sec) & $49.80 \pm 17.82$ & 0.504 \\
TMT-B (sec) & $106.42 \pm 53.43$ & 0.475 \\
TMT-B (errors) & $0.76 \pm 1.00$ & 0.410 \\
TOL (trials solved in minimum moves) & $11.34 \pm 1.02$ & $0.015^{*}$ \\
TOL (instruction failures) & & $\pm .74 \pm 4.10$ & 0.301 \\
TOL (mean of execution time in trials solved) & $6.88 \pm 2.11$ & 0.805 \\
\hline
\end{tabular}

${ }^{\mathrm{a}}$ Adjusted for age, education level and visual acuity

$* p<0.05$

\section{DISCUSSION}

The results of this study support the hypothesis that older adults with driving difficulties have worse results on cognitive tests. Moreover, they are more likely to have lower scores on the Tower of London, a well-known test of executive functioning (particularly planning ability), and worse results on divided attention $\mathrm{UFOV}^{\circledR}$ subtest, reviewed as a valid and reliable index of driving performance and safety (Clay et al., 2005). Considering prospective studies, where this divided-attention task was the best predictor of future crash involvement (Ball et al., 2006; Owsley et al., 1998), we could suppose that this group represents a red flag for crash risk. 
This group of drivers, who demonstrated less ability to plan and solve problems, were probably slightly impaired in supervisory attentional system (SAS) (Norman \& Shallice, 1986), a unitary system related to functioning of the prefrontal cortex and known to decline with age (Salthouse et al., 2003). Some research on older drivers used neuropsychology tests involving the SAS, for instance, TMT-B (Kantor et al., 2004) or a Maze Test (Whelihana et al., 2005), and also indicated a significant relationship between the performance of these tasks and on-road driving performance. In addition, Daigneault et al. (2002) and Ball et al. (2006) found that elderly drivers with a crash history performed poorly on executive measures compared to participants without an accident history. Thus, these higher-order cognitive functions appear to have an important role in driving ability, and our results found them to be a factor related to difficulties in driving. As a result, we expected to find a significant difference between groups in TMT-B scores; however, the difference was slight. It is therefore important to clarify which executive function tests are valid indicators of driving difficulties, how the deterioration of executive functions increases difficulties in driving, and what level of such decline has a predictive value.

There was also an increased risk of driving difficulties associated with female gender. In fact, we cannot be certain if results correspond to actual driving practices or other reasons (e.g., perhaps men tend to overestimate their driving abilities, or women have great facility in expressing their driving difficulties). However, previous research has shown that compared to men, older women drivers report more difficulties (Rimmö \& Hakamies-Blomqvist, 2002), experience more stress in difficult traffic situations (Hakamies-Blomqvist \& Wahlström, 1998) and have higher overall stress levels while driving (Simon \& Corbett, 1996). Thus, there are issues that are specific to the safe mobility of older female drivers and that represent potential for future investigation.

A possible limitation of this study has to do with using self-report as a measure of driving difficulties. As questionnaires are vulnerable to socially desirable response tendencies, it may be that some subjects report fewer driving difficulties than they actually experience. Nevertheless, a study by Lajunen \& Summala (2003) using the Driver Behaviour Questionnaire indicated that the bias caused by socially desirable responding is relatively small, which can help to prove that questionnaires based on self-reports could give a reliable measure relating to driving.

In short, planning ability, divided visual attention and gender seem to be three important factors to consider in the analysis of driving difficulties. However, further investigation with a larger sample of drivers and other additional cognitive tests and driving measures (state-recorded crashes, driving simulator and on-road driving performance) could be developed in order to understand the factors related to driving difficulties in healthy older adults.

\section{ACKNOWLEDGMENTS}

This research is included in a financed project by Fundação para a Ciência e Tecnologia (SFRH/BD/27255/2006).

\section{REFERENCES}

Ball, K., \& Owsley, C. (1993). The Useful Field of View Test: A new technique for evaluating age-related declines in visual function. Journal of the American Optometric Association, 64, 71-79. 
Ball, K., Owsley, C., Stalvey, B., Roenker, D., Sloane, M., \& Graves, M. (1998). Driving avoidance and functional impairment in older drivers. Accident Analysis and Prevention, 30(3), 313-322.

Ball, K., Roenker, D., Wadley, V., Edwards, J., Roth, D., McGwin, G., Raleigh, R., Joyce, J., Cissell, G., \& Dube, T. (2006). Can high-risk older drivers be identified through performance-based measures in a Department of Motor Vehicles setting? Journal of the American Geriatrics Society, 54(1), 77-84.

Ball, K., Vance, D., Edwards, J., \& Wadley, V. (2004). Aging and the brain. In M. Rizzo \& P. Eslinger (Eds.). Principles and practices of behavioral neurology and neuropsychology. Pennsylvania: Elsevier, 795-809.

Clay, O.J., Wadley, V.G., Edwards, J.D., Roth, D.L., Roenker, D.L., Ball., K.K. (2005). Cumulative meta-analysis of the relationship between useful field of view and driving performance in older adults: Current and future implications. Optometry and Visual Science, $82,724-731$.

Daigneault, G., Joly, P., \& Frigon, J-Y. (2002). Executive functions in the evaluation of accident risk of older drivers. Journal of Clinical and Experimental Neuropsychology, 24(2), 221-238.

Folstein, M., Folstein, S., \& McHugh, P. (1975). "Mini-mental state”. A practical method for grading the cognitive state of patients for the clinician. Journal of Psychiatric Research, 12, 189-198.

Hakamies-Blomquist, L., \& Wahlströem, B. (1998). Why do older drivers give up driving? Accident Analysis and Prevention, 30(3), 305-312.

Kantor, B., Mauger, L., Richardson, V., \& Unroe, K. (2004). An analysis of an older driver evaluation program. Journal of the American Geriatrics Society, 52(8), 1326-1330.

Lajunen, T., \& Summala, H. (2003). Can we trust self-reports of driving? Effects of impression management on driver behaviour questionnaire responses. Transportation Research Part F: Traffic Psychology and Behaviour, 6(2), 97-107.

Lezak, M., Howieson, D., \& Loring, D. (2004). Neuropsychological assessment (4 ${ }^{\text {th }}$ ed.). New York: Oxford University Press.

Lundberg, C., Hakamies-Blomqvist, L., Almkvist, O., \& Johansson, K. (1998). Impairments of some cognitive functions are common in crash-involved older drivers. Accident Analysis and Prevention, 30(3), 371-377.

Lundberg, C., Hakamies-Blomqvist, L., Almkvist, O., \& Johansson, K. (2003). License suspension revisited: A 3-year follow-up study of older drivers. The Journal of Applied Gerontology, 22(4), 427-444.

Marottoli, R.A., Cooney, L.M., Wagner, D.R., Doucetter, J., \& Tinetti, M.E. (1994). Predictors of automobile crashes and moving violations among elderly drivers. Annals of Internal Medicine, 121, 842-846.

Maycock, G. (1997). The safety of older car-drivers in the European Union. Report produced for the European Road Safety Federation and the AA Foundation for Road Safety Research. 
Norman, D., \& Shallice, T. (1986). Attention to action: willed and automatic control of behavior. In R. Davidson, G. Schwartz, \& D. Shapiro (Eds), Consciousness and self-regulation. New York: Plenum Press, 1-18.

Owsley, C., Ball, K., McGwin, G., Sloane, M., Roenker, D., White, M., \& Overley, E. (1998). Visual processing impairment and risk of motor vehicle crash among older adults. Journal of the American Medical Association, 279(14), 1083-1088.

Owsley, C., Stalvey, B., Wells, J., \& Sloane, M. (1999). Older drivers and cataract: Driving habits and crash risk. Journals of Gerontology Series A: Biological Sciences and Medical Sciences, 54A(4), M203-M211.

Reitan, R. (1958). Validity of the Trail Making test as an indicator of brain damage. Perceptual and Motor Skills, 8, 271-276.

Rey, A. (1941). L'examen psychologique dans les cas d'encéphalopathie traumatique. Archives de Psychologie, 28, 286-340.

Richardson, E., \& Marottoli, R. (2003). Visual attention and driving behaviors among community-living older persons. Journals of Gerontology Series A: Biological and Medical Sciences, 58(9), M832-M836.

Rimmö, P.-A., \& Hakamies-Blomqvist, L. (2002). Older drivers' aberrant driving behaviour, impaired activity, and health as reasons for self-imposed driving limitations. Transportation Research. Part F: Traffic Psychology and Behaviour, 5(1), 47-62.

Salthouse, T.A., Atkinson, T.M., \& Berish, D.E. (2003). Executive functioning as a potential mediator of age-related cognitive decline in normal adults. Journal of Experimental Psychology \& Genetics, 132, 566-594.

Shallice, T. (1982). Specific impairments of planning. Philosophical transcripts of the Royal Society of London, 298, 199-290.

Simon, F., \& Corbett, C. (1996). Road traffic offending, stress, age, and accident history among male and female drivers. Ergonomics, 39, 757-780.

Strauss, A., Sherman, E., \& Spreen, O. (2006). Compendium of neuropsychological tests: Administration, norms, and commentary ( $3^{\text {rd }}$ ed.). New York: Oxford University Press.

Spreen, O. \& Strauss, E. (1998). A compendium of neuropsychological tests: Administration, norms, and commentary ( $2^{\text {nd }}$ ed.). New York: Oxford University Press.

Wechsler (1997). Wechsler Adult Intelligence Scale - Third Edition. San Antonio, TX: The Psychological Corporation.

Whelihana, W., DiCarlo, M., \& Paul, R. (2005). The relationship of neuropsychological functioning to driving competence in older persons with early cognitive decline. Archives of Clinical Neuropsychology, 20, 217-228.

Wild, K., \& Cottrell, V. (2003). Identifying driving impairment in Alzheimer disease: A comparison of self and observer reports versus driving evaluation. Alzheimer Disease and Associated Disorders, 17, 27-34. 